\title{
In situ evidence of resonant interactions between energetic electrons and whistler waves in magnetopause reconnection
}

\author{
Zhi Li ${ }^{1,2}$, QuanMing Lu1,2*, RongSheng Wang, XinLiang Gao, and HuaYue Chen ${ }^{1,2}$ \\ ${ }^{1}$ Chinese Academy Sciences Key Laboratory of Geospace Environment, Department of Geophysics and Planetary Science, University of Science and \\ Technology of China, Hefei 230026, China; \\ ${ }^{2}$ Chinese Academy Sciences Center for Excellence in Comparative Planetology, Hefei 230026, China
}

\begin{abstract}
In this paper, we analyze one reconnection event observed by the Magnetospheric Multiscale (MMS) mission at the earth's magnetopause. In this event, the spacecraft crossed the reconnection current sheet from the magnetospheric side to the magnetosheath side, and whistler waves were observed on both the magnetospheric and magnetosheath sides. On the magnetospheric side, the whistler waves propagated quasi-parallel to the magnetic field and toward the X-line, while on the magnetosheath side they propagated almost anti-parallel to the magnetic field and away from the X-line. Associated with the enhancement of the whistler waves, we find that the fluxes of energetic electrons are concentrated around the pitch angle $90^{\circ}$ when their energies are higher than the minimum energy that is necessary for the resonant interactions between the energetic electrons and whistler waves. This observation provides in situ observational evidence of resonant interactions between energetic electrons and whistler waves in the magnetic reconnection.
\end{abstract}

Keywords: magnetic reconnection; whistler waves; magnetosphere; energetic electrons

Citation: Li, Z., Lu, Q. M., Wang, R. S., Gao, X. L., and Chen, H. Y. (2019). In situ evidence of resonant interactions between energetic electrons and whistler waves in magnetopause reconnection. Earth Planet. Phys., 3(6), 467-473. http://doi.org/10.26464/epp2019048

\section{Introduction}

Magnetic reconnection is a fundamental physical process occurring when two plasmas with different magnetic field topologies interact to change the magnetic structure. This process can efficiently convert magnetic energy into plasma kinetic energy (Priest and Forbes, 2000; Fu HS et al., 2015; Treumann and Baumjohann, 2015; Yamada et al., 2010; Lu S et al., 2019), and is considered to be responsible for explosive energy releases in space environments, such as solar flares (Ciaravella and Raymond, 2008) and substorms in the earth's magnetosphere (Russell and McPherron, 1973). During magnetic reconnection, the magnetic field lines reconnect in a small region called the diffusion region, which includes both the ion diffusion region on the ion inertial length scale and the electron diffusion region on the electron inertial length scale (Birn et al., 2001; Lu QM et al., 2010). In the ion diffusion region, electrons are frozen in the magnetic field and ions can cross the magnetic field lines. In the electron diffusion region (EDR), both ions and electrons are unmagnetized. Magnetic reconnection is considered to be initiated in the electron diffusion region and can then be extended to a large scale (Vasyliunas et al., 1975).

Various types of plasma waves have been found to be associated with magnetic reconnection. Plasma waves can transfer mo-

Correspondence to: Q. M. Lu, qmlu@ustc.edu.cn

Received 28 JAN 2019; Accepted 20 AUG 2019.

Accepted article online 12 SEP 2019.

C 2019 by Earth and Planetary Physics. mentum and energy among particles, which results in anomalous resistivity and formation of energetic particles (Ji et al., 2004, Zhou et al., 2014). Among these plasma wave, whistler waves have received special attention. The first in situ observational evidence of whistler waves was identified by Deng XH and Matsumoto (2001) in the diffusion region of a magnetopause reconnection event, and then Wei et al. (2007) found enhancement of whistler waves in Cluster observations made during a magnetotail reconnection event. Particle-in-cell simulation of anti-parallel reconnection revealed that whistler waves can be excited in the downstream of the electron outflow by electron temperature anisotropy (Fujimoto and Sydora, 2008) and in the separatrix region by an electron beam (Fujimoto, 2014). With Cluster observations, Huang SY et al. $(2016,2017)$ confirmed the existence of whistler waves in both the downstream of the electron outflow and the separatrix region by statistically analyzing the reconnection events in the magnetotail. Characteristics of whistler waves in asymmetric reconnection have also been thoroughly studied when satellites cross the magnetopause. In asymmetric reconnection, whistler waves have been observed in the separatrix region (Graham et al., 2016; Wilder et al., 2016; Li et al., 2018; Burch et al., 2018), as well as in the electron diffusion region (Tang XW et al., 2013; Cao D et al., 2017). Recently, with the launching of the Magnetospheric Multiscale (MMS) mission, taking advantage of its high resolution electron plasma data, Cao D et al. (2017) indicated that the whistler waves observed in magnetic reconnection are excited by electron temperature anisotropy. In this paper, based on MMS mission observations of a magnetopause reconnection event, we analyze in detail the distributions of pitch angles of energetic 
electrons at different energies. Our findings provide in situ evidence of resonant interactions of energetic electrons and whistler waves in the separatrix regions of both the magnetospheric and the magnetosheath sides.

\section{Observations of a Magnetopause Reconnection}

\section{Event}

NASA's MMS mission was launched in March 2015; its main goal is to study magnetic reconnection on the electron scale (Burch et al., 2016). The mission includes four spacecraft in an approximate tetrahedral formation. Each spacecraft measures particle data, electric field, and so on, at high temporal resolution. The present study uses measurements of the electric and magnetic fields and wave and particle data from the comprehensive locations of instruments onboard the MMS spacecraft (Burch et al., 2016). The magnetic field is sampled by Fluxgate Magnetometers at 128/s in the burst mode and $16 / \mathrm{s}$ in the fast survey mode (Russell et al., 2016, Torbert et al., 2016). The electric field is sampled by Elecctric Double Probes at $8192 / \mathrm{s}$ in the burst mode and $32 / \mathrm{s}$ in the fast survey mode (Ergun et al., 2016, Lindqvist et al., 2016). The tem- poral resolutions of electron and ion data measured by the Fast Plasma Instrument are $30 \mathrm{~ms}$ and $150 \mathrm{~ms}$, respectively (Pollock et al., 2016). The ion and electron distributions electrons with $4.5 \mathrm{~s}$ resolution in the fast survey mode are also provided by Fast Plasma Instruments. The energy range of the Fast Plasma Instrument is from $10 \mathrm{eV}$ to $30 \mathrm{keV}$ for both electrons and ions. The three magnetic components of waves are measured at $8192 / \mathrm{s}$ by Search Coil Magnetometers and are used to study whistler wave characteristics (Le Contel et al., 2016).

A magnetic reconnection event was observed by MMS from 05:56:44 to 05:57:11 UT on 12 November 2015, when the spacecraft was located at $[11.3,1.7,-1.3]$ Earth radius $\left(R_{\mathrm{E}}\right)$ in the geocentric solar magnetospheric (GSM) coordinate system. Since the spacecraft separation was about $20 \mathrm{~km}$ during this time period, observations of this event at all four spacecraft were similar; we use only data from MMS3.

Figures $1(\mathrm{a}-\mathrm{h})$ show an overview of this magnetopause reconnection event. The data are given in a local current sheet (LMN) coordinate system, which is determined from Minimum Variance Analysis (Sonnerup and Scheible, 1998) applied to the ambient

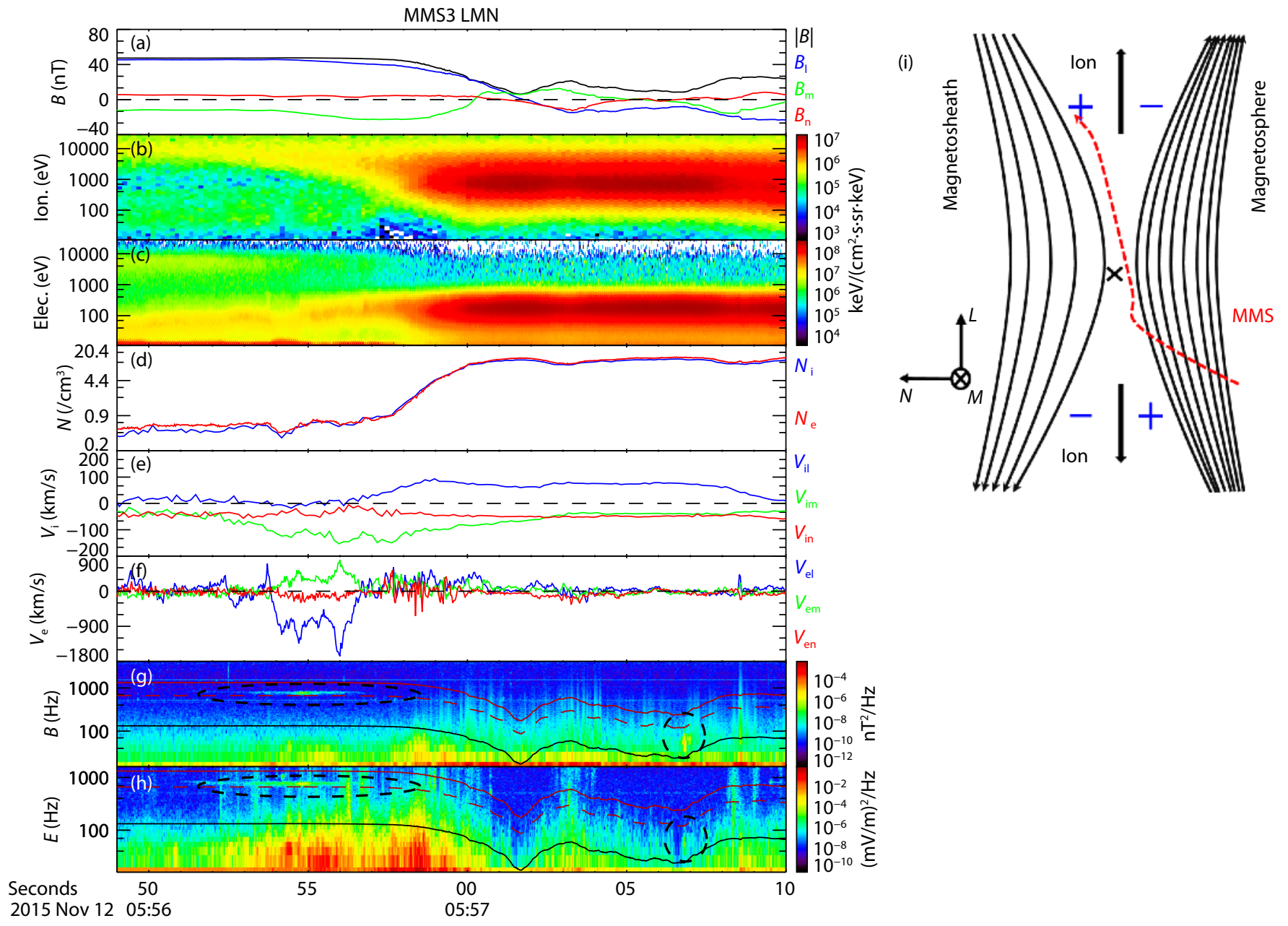

Figure 1. Overview of MMS3 observations in the burst mode during 05:56:44-05:57:11 UT on 12 November 2015. (a) Magnetic field vector in the LMN coordinate, $(b-c)$ ion and electron energy spectra, $(d)$ ion and electron number density, $(e)$ ion bulk velocity in the LMN coordinate, ( $f$ ) electron bulk velocity in the LMN coordinate, $(\mathrm{g}-\mathrm{h})$ magnetic and electric field power spectral density; the black solid lines, red dashed lines, and red solid lines correspond to $0.1,0.5$, and 1.0 of the local electron gyrofrequency, (i) spacecraft trajectory crossing the magnetopause event. 
magnetic field during the current sheet crossing (05:56:53-05:57:00 UT). With respect to the GSM coordinates, $M=(-0.207,0.978,-0.015)$ mainly pointing to the dusk, $N=(-0.933,-0.193,0.302)$ is the boundary normal direction and almost identical to the $X$-axis in the GSM coordinates, and $L=(0.293,0.076,0.953)$ completes the right-hand system.

In the beginning, the spacecraft was in the magnetosphere, identified by the stable and northward $B_{\mathrm{L}}(\sim 58 \mathrm{nT}$, Figure $1 \mathrm{a})$, and lowdensity plasma ( $<1 \mathrm{~cm}^{-3}$, Figure $1 \mathrm{~d}$ ). From 05:56:54 to 05:56:57 UT, the electrons have a larger bulk velocity in the $L$ direction, reaching a maximum value of $-1600 \mathrm{~km} / \mathrm{s}$ (Figure $1 \mathrm{f}$ ) in the outflow region. We also find that both the electrons and ions have an energetic component, of approximately $0.3 \mathrm{keV}$ and $1.0 \mathrm{keV}$, respectively. These energetic components are generated during magnetic reconnection. At approximately 05:56:57.1 UT, MMS3 entered the magnetosheath, where the plasma density was high and the magnetic field $B_{\mathrm{L}}$ turned from northward to southward. The ions obtained a large bulk velocity in the $L$ direction, and the spacecraft was in the northward outflow region. The spacecraft trajectory crossing the magnetopause reconnection event is plotted in Figure $1 \mathrm{i}$. In this reconnection event, we can also observe high-frequency electromagnetic waves on both the magnetosphere and magnetosheath sides, circled by dashed lines in Figures $1 \mathrm{~g}$ and 1h. Their frequencies are around 760 and $65 \mathrm{~Hz}$, respectively, which are about 0.56 and 0.2 of the local electron gyrofrequency.

Figure 2 presents the characteristics of these electromagnetic waves. These wave characteristics are analyzed in the fieldaligned coordinates (FAC) system, which is described as follows: the $Z$-axis is parallel to the mean magnetic field measured by the

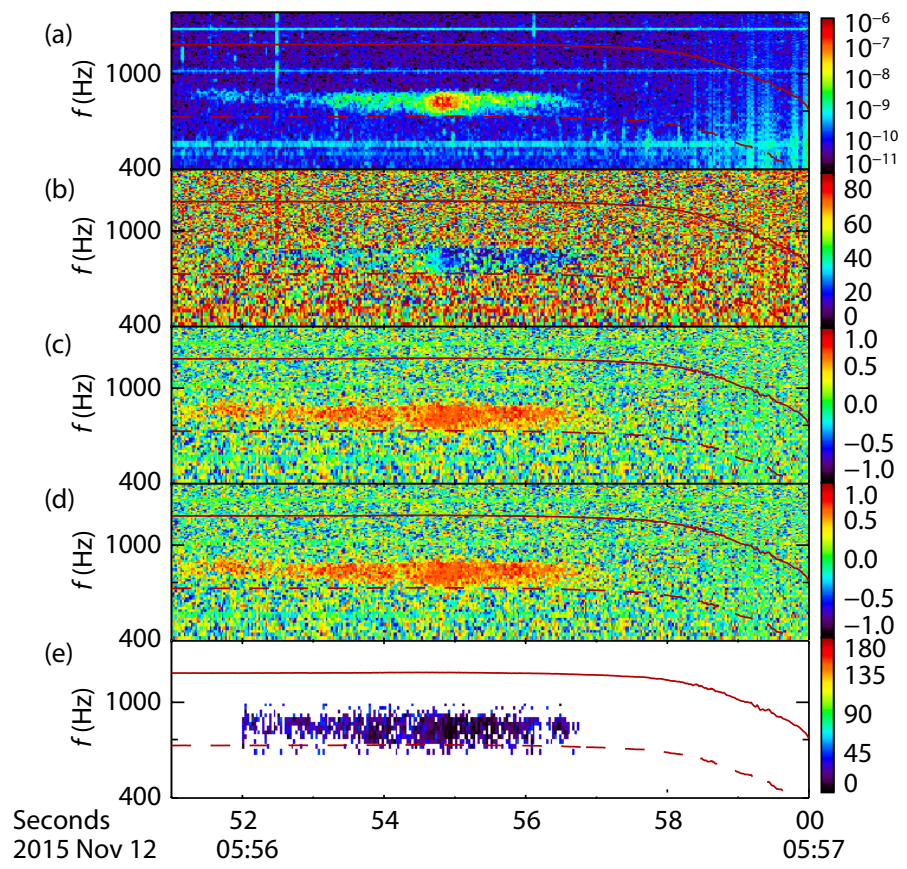

Fluxgate Magnetometer at the burst data rate (128/s). The $X$-axis is perpendicular to the mean magnetic field and in the spacecraft spin plane, and the $Y$-axis completes the right-hand system. The polarization analysis is obtained from the spectral matrix computed using SCM waveforms (Samson and Olson, 1980), where the wave normal angle, the ellipticity, and the helicity are resolved. According to this method, the ellipticity and helicity of the right-hand polarized waves are equal to 1 , and the waves have left-handed polarization when the ellipticity and helicity are equal to -1 . Figures 2(a-e) plot the frequencies, wave normal angles, ellipticities, helicities, and angles between the Poynting fluxes and the magnetic field of the plasma waves on the magnetospheric sides. From about 05:56:53 UT to 05:56:57 UT, the enhancement of the plasma waves can be observed. Analysis of the magnetic spectra shows that the frequencies of the waves are about 0.56 of the local electron gyrofrequency. The ellipticity and helicity (Figures 2(c-d)) are approximately 1.0, and the magnetospheric waves have right-hand polarization. Their wave normal angle is approximately $25^{\circ}$. Therefore, the observed electromagnetic waves on the magnetospheric sides are whistler waves. From the angle between the Poynting flux and magnetic field (Figure 2e), we conclude that the propagating angle of the magnetospheric waves is about $22^{\circ}$, and they propagate toward the $\mathrm{X}$-line.

Figures $2(f-j)$ present the frequencies, wave number angles, ellipticities, helicities, and angles between the Poynting fluxes and magnetic field of the magnetosheath waves. We can observe obvious enhancement of plasma waves from 05:57:06.7 UT to 05:57:07 UT. Analysis of magnetic spectra shows that waves with frequencies from 0.1 and 0.5 of the local electron gyrofrequency are right-hand circularly polarized. The wave normal angle is

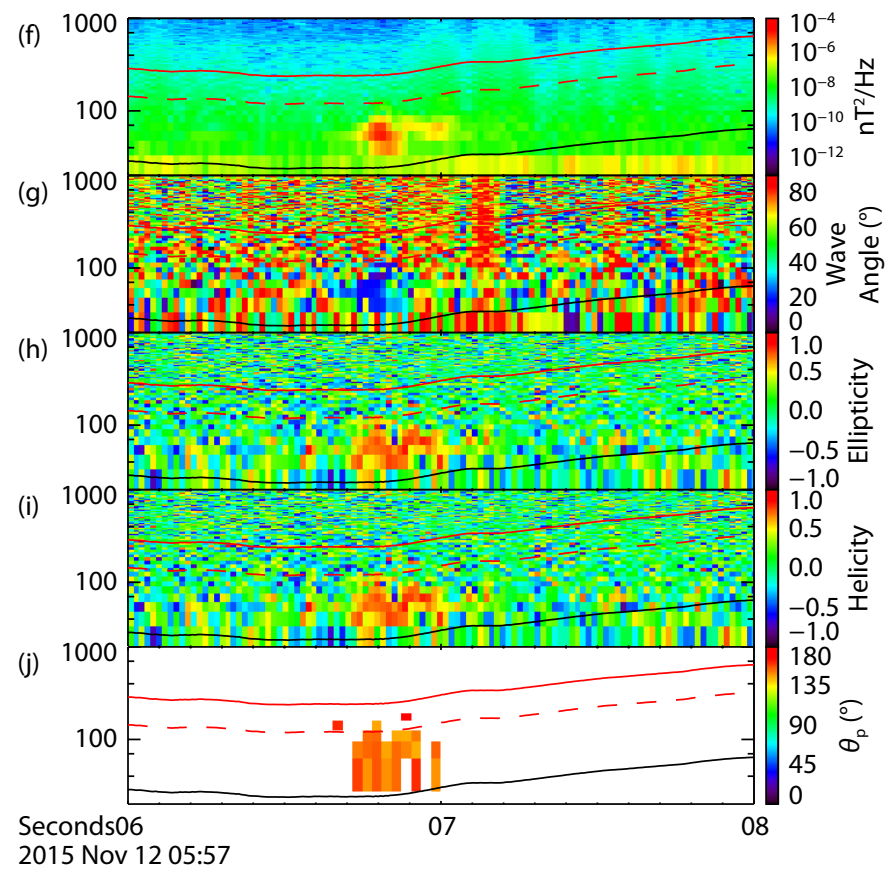

Figure 2. Characteristics of the electromagnetic waves on magnetospheric and magnetosheath sides. (a)-(e) plot characteristics of magnetospheric waves: (a) magnetic field power spectrogram, (b) wave angle, (c) ellipticity (+1 denotes right-handed circular polarization, -1 denotes left-handed circular polarization), (d) helicity similar to ellipticity and (e) the angle between the wave Poynting flux and magnetic field. (f)-(j) plot the corresponding characteristics of magnetosheath waves. 
about $20^{\circ}$, and the waves propagate in a direction quasi-parallel to the magnetic field. All these features suggest that these wave trains are also whistler waves. From the angle between the Poynting fluxes and magnetic field (Figures $2 \mathrm{j}$ ), we can conclude that the propagating angle of the magnetospheric waves is $165^{\circ}$, which means that magnetosheath waves are almost antiparallel to the magnetic field. Since the reconnection site is determined to be located at the southward of the spacecraft on the magnetosheath side, the magnetosheath waves propagate away from the $\mathrm{X}$-line.

Figure 3 illustrates the time evolution of the electron differential fluxes (from 798 to $4000 \mathrm{eV}$ ) at different pitch angles from 05:56:53.8 to 05:56:58.8 UT, when the spacecraft was on the magnetospheric side. As shown in Figure 2, the electromagnetic waves begin to be enhanced at about 05:56:53 UT on the magnetospheric side. We find that these waves can scatter electrons with energy higher than about $1024 \mathrm{eV}$, and the pitch angles of these electrons are concentrated around $90^{\circ}$. This is seen more clearly in Figure 4, which shows the electron energy fluxes in the parallel, anti-parallel, and perpendicular directions at 05:56:51.7 UT (a) and 05:56:55.1 UT (b). Consistent with Figure 3, with the enhancement of the whistler waves (Figure 4b), the energy flux in the perpendicular direction is much larger than fluxes in the parallel and anti-parallel directions.
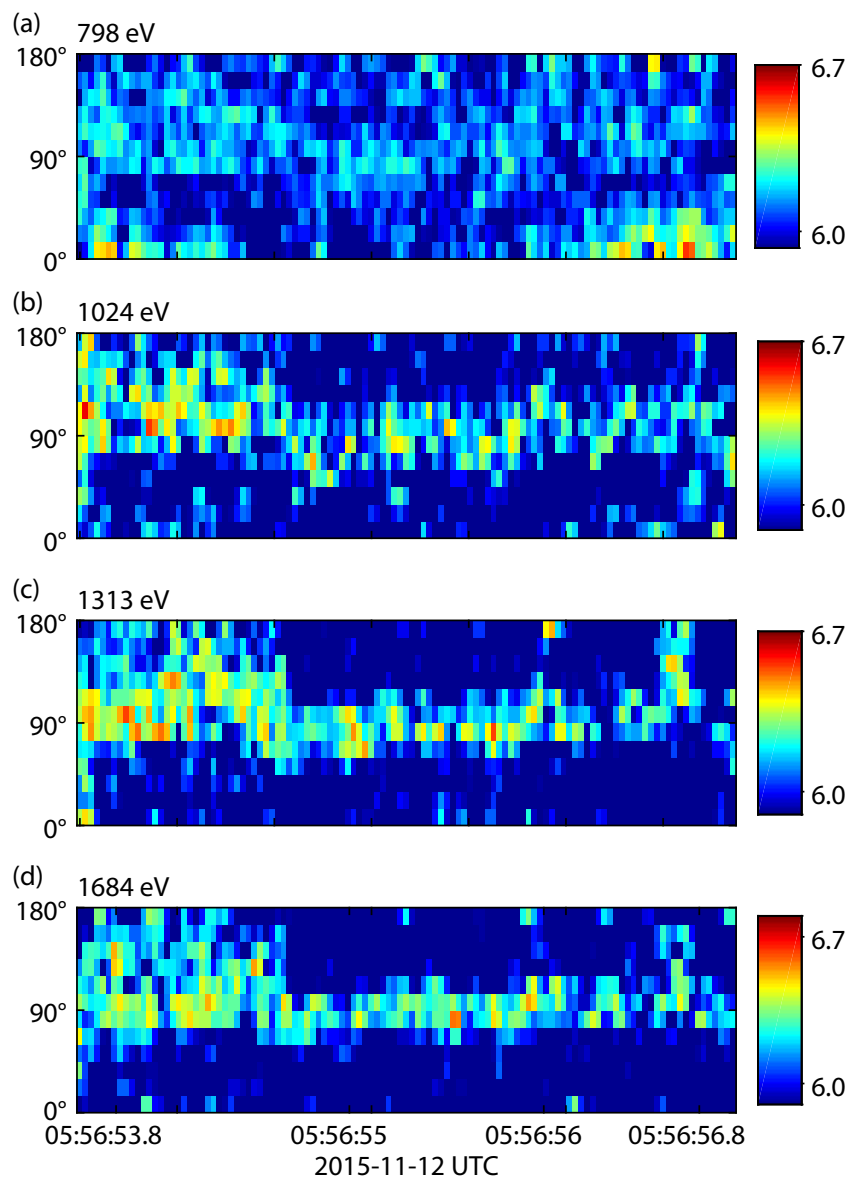

Figure 5 plots the time evolution of the electron differential fluxes (from 52 to $1024 \mathrm{eV}$ ) at different pitch angles from 05:57:06.2 to 05:57:07.1 UT, when the spacecraft was on the magnetosheath side. As shown in Figure 2, the electromagnetic waves begin to be enhanced at about 05:57:06.7 UT on the magnetospheric side. We note that when the electron energy is less than about $140 \mathrm{eV}$, the pitch angles of electrons are concentrated around $0^{\circ}$ and $180^{\circ}$. However, for electrons with energy higher than $140 \mathrm{eV}$, their pitch angles begin to be concentrated around $90^{\circ}$ with the enhancement of the whistler waves. This pattern can be also be identified in Figure 6, which plots electron energy fluxes in the parallel, antiparallel and perpendicular directions at 05:57:06.1 UT (a) and 05:57:06.73 UT (b). It is clear that the enhanced whistler waves can scatter electrons with energy higher than about $140 \mathrm{eV}$, and that the energy flux in the perpendicular direction is much larger than fluxes in the parallel and anti-parallel directions.

\section{Discussion and Conclusions}

Based on Faraday equation in Fourier space, we can obtain

$$
\boldsymbol{k} \times \delta \boldsymbol{E}(\omega, \boldsymbol{k})=\omega \delta \boldsymbol{B}(\omega, \boldsymbol{k}),
$$

where $\delta \boldsymbol{E}(\omega, \boldsymbol{k})$ and $\delta \boldsymbol{B}(\omega, \boldsymbol{k})$ are the electric and magnetic fluctuations in Fourier space, obtained by fast Fourier transformation of the electric and magnetic field data. If the plasma waves have a dominant wave mode, such as the observed whistler waves in this (e) $2159 \mathrm{eV}$

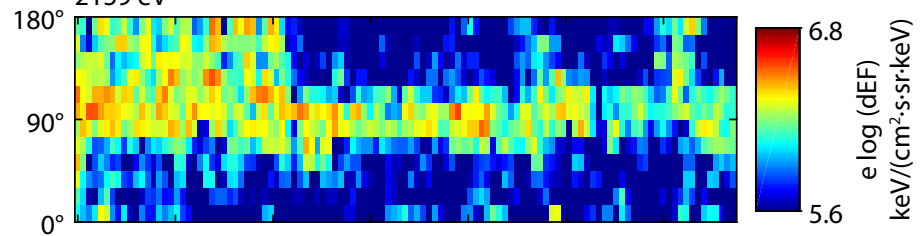

(f) $2950 \mathrm{eV}$

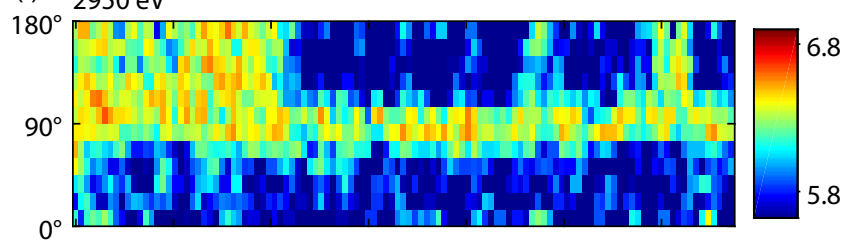

(g) $3850 \mathrm{eV}$

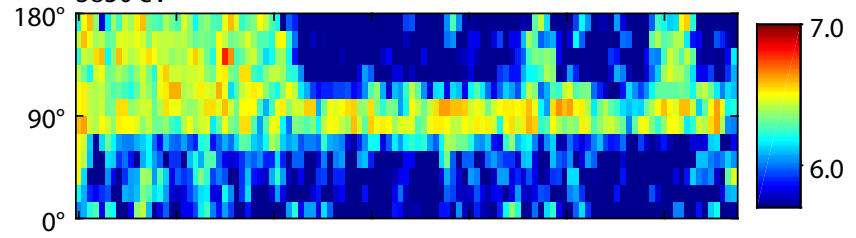

(h) $4000^{+} \mathrm{eV}$

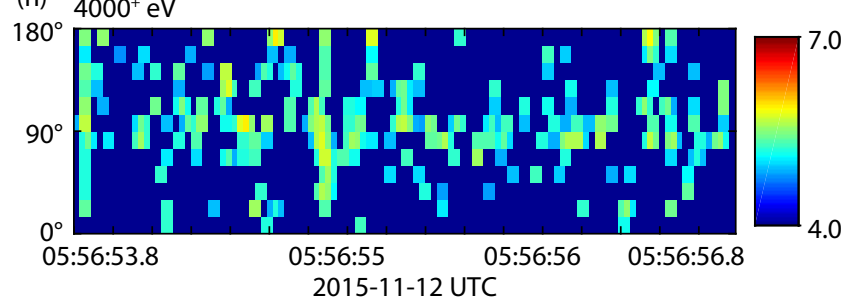

Figure 3. Time evolution of the electron differential energy fluxes (dEF) (from 798 to $4000 \mathrm{eV}$ ) at different pitch angles, from 05:56:53.8 to 05:56:58.8 UT, when the spacecraft was on the magnetospheric side. 

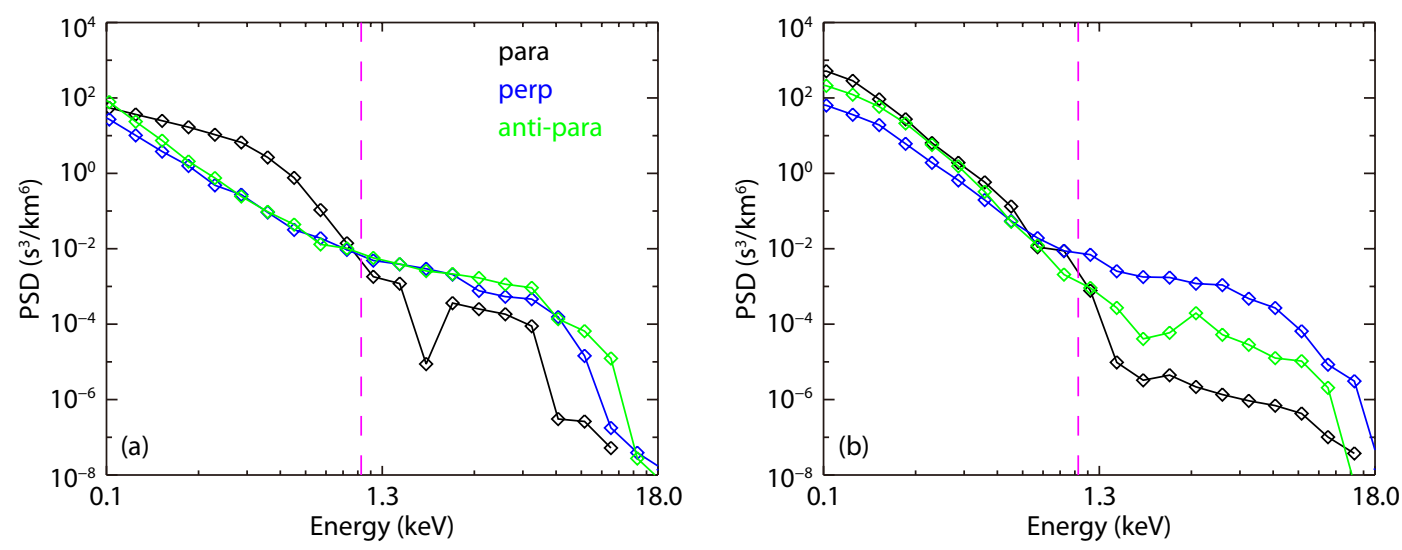

Figure 4. Electron energy fluxes in the parallel, anti-parallel and perpendicular directions at (a) 05:56:51.7 UT and (b) 05:56:55.1 UT, when the spacecraft was on the magnetospheric side. The vertical lines correspond to electrons with at least $1100 \mathrm{eV}$.
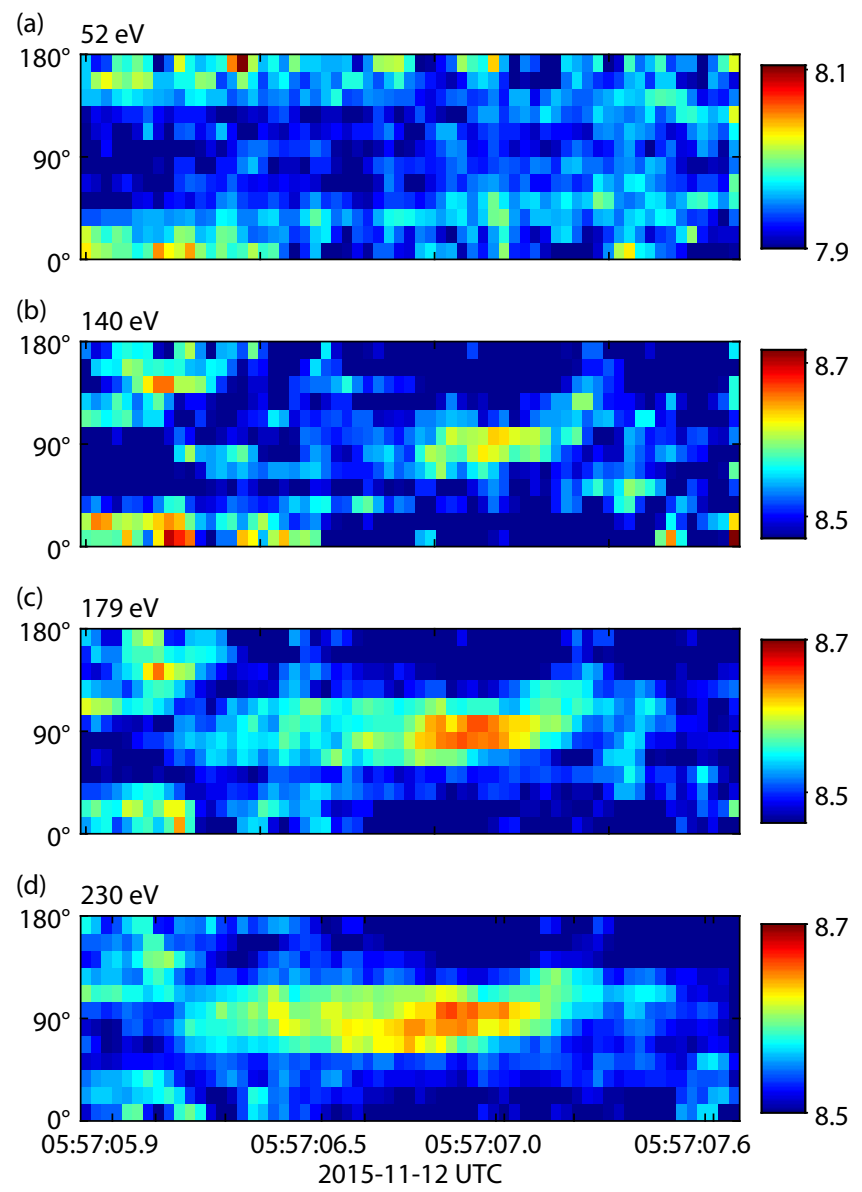
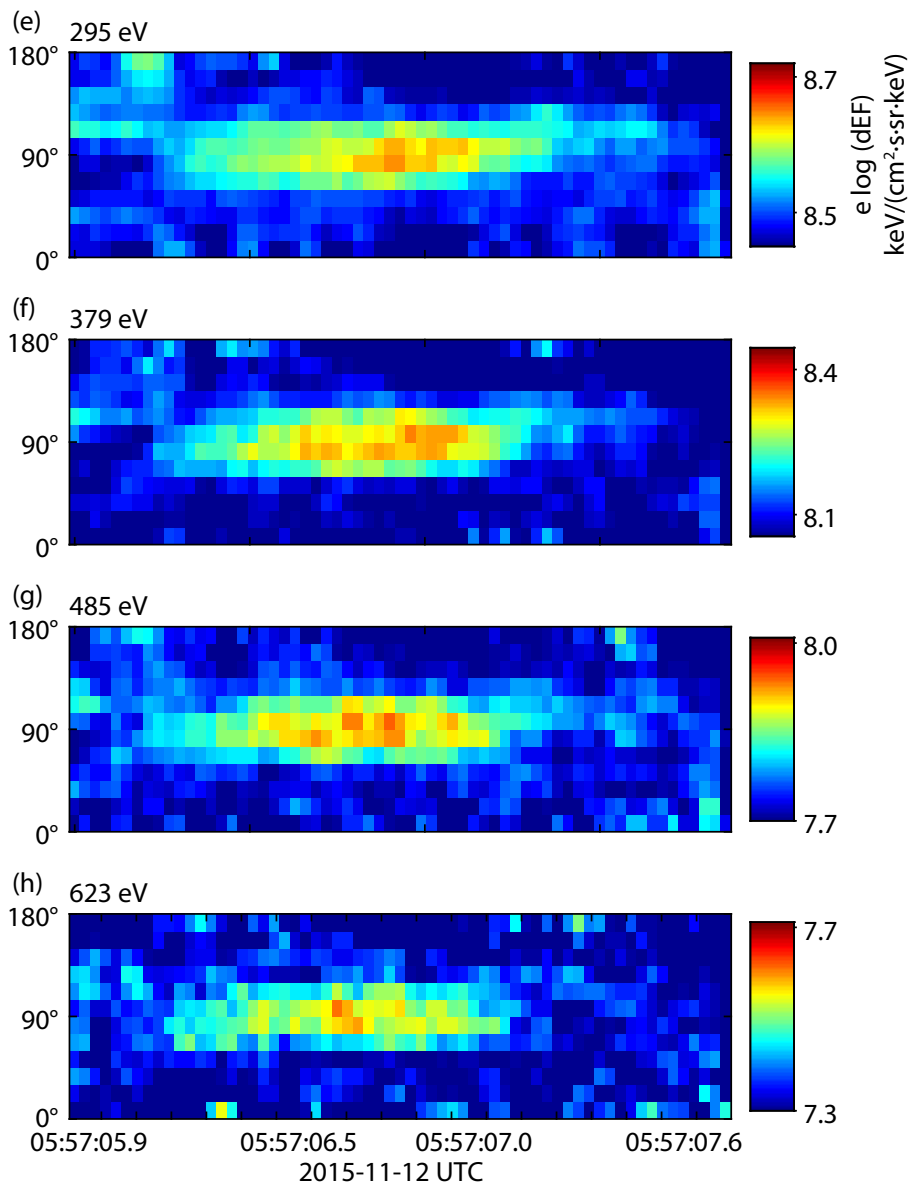

Figure 5. Time evolution of the electron differential energy fluxes (dEF) (from 52 to $1024 \mathrm{eV}$ ) at different pitch angles from 05:57:06.2 to 05.57.07.1 UT, when the spacecraft was on the magnetosheath side.

event, we can calculate the corresponding wave number $\boldsymbol{k}$ according to Equation (1). Then, after the wave normal angle is determined, we can estimate the resonant velocity $v_{\|}$based on the cyclotron resonant condition $\omega-k_{\| \mid} v_{\|}= \pm 2 \pi f_{c e}$ (where $f_{\mathrm{ce}}=e B /\left(2 \pi m_{\mathrm{e}}\right)$ is the electron gyrofrequency, and + and - are used for right- and left-hand polarized waves, respectively). The minimum energy of the electrons that can be scattered by the electromagnetic waves is $\varepsilon_{\min }=0.5 m_{\mathrm{e}} v_{\|}^{2}$ (Kennel and Petschek,
1966). In other words, only electrons with energy higher than $\varepsilon_{\min }$ can resonantly interact with the electromagnetic waves.

In this reconnection event in the magnetopause, we observe whistler waves on both the magnetospheric and magnetosheath sides. On the magnetospheric side, according to Equation (1), we calculate the wave number of the dominant whistler wave mode to be $k_{\|}=3 \times 10^{-5} \mathrm{~m}^{-1}$. It follows that the minimum energy of electrons able to interact resonantly with the whistler waves is ap- 

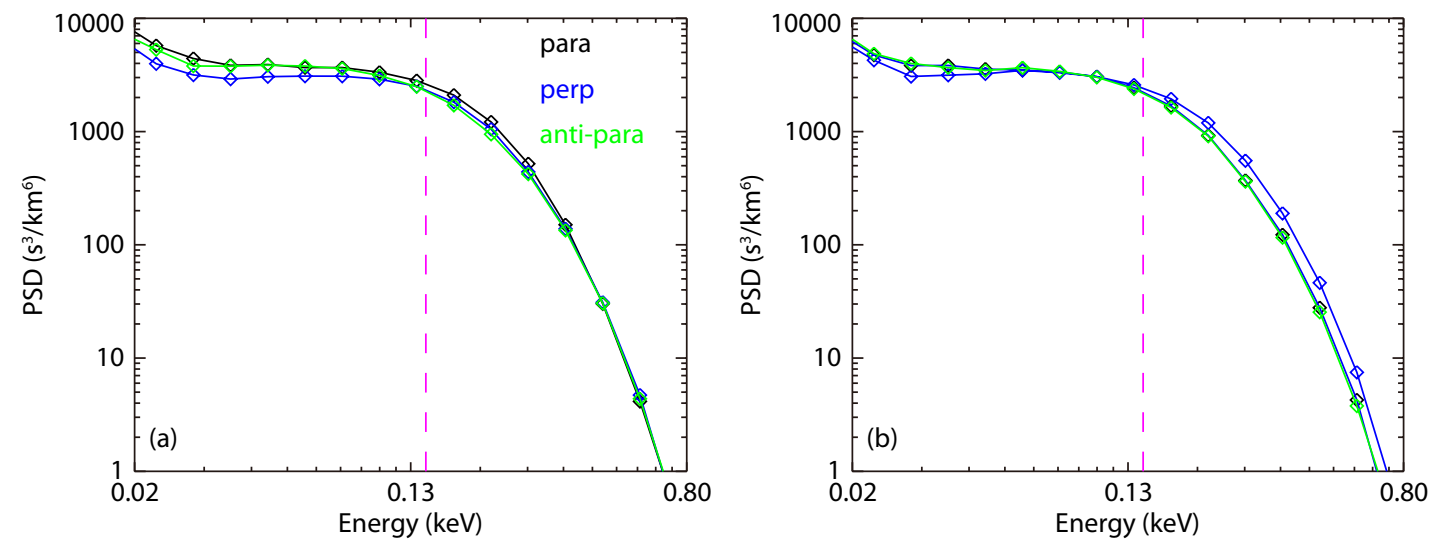

Figure 6. Electron energy fluxes in the parallel, anti-parallel, and perpendicular directions at (a) 05:57:06.1 UT and (b) 05:57:06.73 UT, when the spacecraft was on the magnetospheric side. The vertical lines correspond to the electrons with at least $142 \mathrm{eV}$.

proximately $1100 \mathrm{eV}$. As described in Figure 3, associated with the enhancement of the whistler waves between 05:56:52 UT to 05:56:57 UT, the electron fluxes with energy higher than $1100 \mathrm{eV}$ increase at pitch angles close to $90^{\circ}$. We attribute this observation to resonant interactions with the whistler waves.

On the magnetosheath side, we calculate the wave number of the dominant whistler mode wave to be $k_{\|}=1 \times 10^{-5} \mathrm{~m}^{-1}$, and the minimum energy of the electrons scattered by the whistler waves to be about $142 \mathrm{eV}$. This is consistent with the demonstration of Figure 5 that the electrons with energy higher than $142 \mathrm{eV}$ can resonantly interact with the whistler waves and exhibit pitch angles concentrated around $90^{\circ}$.

In summary, by analyzing a magnetopause reconnection event, we identify whistler waves on both the magnetospheric and magnetosheath sides. Associated with these whistler waves, fluxes of electrons with energies higher than the minimum energy are enhanced around the $90^{\circ}$ pitch angle through resonant interactions with the whistler waves. This analysis provides in situ observational evidence for resonant interactions between energetic electrons and whistler waves.

\section{Acknowledgments}

This work was supported by NSFC grants 41527804 and 41774169, and by Key Research Program of Frontier Sciences, CAS(QYZDJ-SSW-DQC010).

\section{References}

Birn, J., Drake, J. F., Shay, M. A., Rogers, B. N., Denton, R. E., Hesse, M., Kuznetsova, M., Ma, Z. W., Bhattacharjee, A., ... Pritchett, P. L. (2001). Geospace Environmental Modeling (GEM) magnetic reconnection challenge. J. Geophys. Res. Space Phys., 106(A3), 3715-3719. https://doi.org/10.1029/1999JA900449

Burch, J. L., Moore, T. E., Torbert, R. B., and Giles, B. L. (2016). Magnetospheric multiscale overview and science objectives. Space Sci. Rev., 199(1-4), 5-21. https://doi.org/10.1007/s11214-015-0164-9

Burch, J. L., Ergun, R. E., Cassak, P. A., Webster, J. M., Torbert, R. B., Giles, B. L., Dorelli, J. C., Rager, A. C., Hwang, K. J., ... Newman, D. L. (2018). Localized oscillatory energy conversion in magnetopause reconnection. Geophys. Res. Lett., 45(3), 1237-1245. https://doi.org/10.1002/2017GL076809

Cao, D., Fu, H. S., Cao, J. B., Wang, T. Y., Graham, D. B., Chen, Z. Z., Peng, F. Z., Huang, S. Y., Khotyaintsev, Y. V., ... Burch, J. L. (2017). MMS observations of whistler waves in electron diffusion region. Geophys. Res. Lett., 44(9), 3954-3962. https://doi.org/10.1002/2017GL072703

Ciaravella, A., and Raymond, J. C. (2008). The current sheet associated with the 2003 November 4 coronal mass ejection: density, temperature, thickness, and line width. Astrophys. J., 686(2), 1372-1382. https://doi.org/10.1086/590655

Deng, X. H., and Matsumoto, H. (2001). Rapid magnetic reconnection in the Earth's magnetosphere mediated by whistler waves. Nature, 410(6828), 557-560. https://doi.org/10.1038/35069018

Ergun, R. E., Tucker, S., Westfall, J., Goodrich, K. A., Malaspina, D. M., Summers, D., Wallace, J., Karlsson, M., Mack, J., ... Rau, D. (2016). The Axial Double Probe and fields signal processing for the MMS mission. Space Sci. Rev., 199(1-4), 167-188. https://doi.org/10.1007/s11214-014-0115-x

Fu, H. S., Vaivads, A., Khotyaintsev, Y. V., Olshevsky, V., André, M., Cao, J. B., Huang, S. Y., Retinò, A., and Lapenta, G. (2015). How to find magnetic nulls and reconstruct field topology with MMS data?. J. Geophys. Res. Space Phys., 120(5), 3758-3782. https://doi.org/10.1002/2015JA021082

Fujimoto, K., and Sydora, R. D. (2008). Whistler waves associated with magnetic reconnection. Geophys. Res. Lett., 35(19), L19112. https://doi.org/10.1029/2008GL035201

Fujimoto, K. (2014). Wave activities in separatrix regions of magnetic reconnection. Geophys. Res. Lett., 41(8), 2721-2728. https://doi.org/10.1002/2014GL059893

Graham, D. B., Vaivads, A., Khotyaintsev, Y. V., and André, M. (2016). Whistler emission in the separatrix regions of asymmetric magnetic reconnection. J. Geophys. Res. Space Phys., 121(3), 1934-1954. https://doi.org/10.1002/2015JA021239

Huang, S. Y., Fu, H. S., Yuan, Z. G., Vaivads, A., Khotyaintsev, Y. V., Retino, A., Zhou, M., Graham, D. B., Fujimoto, K., ... Zhou, X. (2016). Two types of whistler waves in the hall reconnection region. J. Geophys. Res. Space Phys., 121(7), 6639-6646. https://doi.org/10.1002/2016JA022650

Huang, S. Y., Yuan, Z. G., Sahraoui, F., Fu, H. S., Pang, Y., Zhou, M., Fujimoto, K., Deng, X. H., Retinò, A., ... Li, H. M. (2017). Occurrence rate of whistler waves in the magnetotail reconnection region. J. Geophys. Res. Space Phys., 122(7), 7188-7196. https://doi.org/10.1002/2016JA023670

Ji, H., Terry, S., Yamada, M., Kulsrud, R., Kuritsyn, A., and Ren Y. (2004). Electromagnetic fluctuations during fast reconnection in a laboratory plasma. Phys. Rev. Lett., 92, 115001 https://doi.org/10.1103/PhysRevLett.92.115001

Kennel, C. F., and Petschek, H. E. (1966). Limit on stably trapped particle fluxes. J. Geophys. Res., 71(1), 1-28. https://doi.org/10.1029/JZ071i001p00001

Le Contel, O., Leroy, P., Roux, A., Coillot, C., Alison, D., Bouabdellah, A., Mirioni, L., Meslier, L., Galic, A., ... de la Porte, B. (2016). The search-coil magnetometer for MMS. Space Sci. Rev., 199(1-4), 257-282. https://doi.org/10.1007/s11214-014-0096-9

Li, J., Bortnik, J., An, X., Li, W., Russell, C. T., Zhou, M., and Le Contel, O. (2018). Local excitation of whistler mode waves and associated Langmuir waves at 
dayside reconnection regions. Geophys. Res. Lett., 45(17), 8793-8802. https://doi.org/10.1029/2018GL078287

Lindqvist, P. A., Olsson, G., Torbert, R. B., King, B., Granoff, M., Rau, D., Needell, G. Turco, S., Dors, I., ... Tucker, S. (2016). The spin-plane double probe electric field instrument for MMS. Space Sci. Rev., 199(1-4), 137-165. https://doi.org/10.1007/s11214-014-0116-9

Lu, Q. M., Huang, C., Xie, J. L., Wang, R. S., Wu, M. Y., Vaivads, A., and Wang, S. (2010). Features of separatrix regions in magnetic reconnection: Comparison of 2-D particle-in-cell simulations and Cluster observations. J. Geophys. Res.: Space Phys., 115(A11), A11208. https://doi.org/10.1029/2010JA015713

Lu, S., Angelopoulos, V., Artemyev, A. V., Pritchett, P. L., Runov, A., Tenerani, A., Shi., C., Velli, M. (2019). Turbulent and particle acceleration in collisionless magnetic reconnection: Effects of temperature inhomogeneity across preconnection current sheet. Astrophys. J., 878, 109. https://doi.org/10.3847/1538-4357/ab1f6b

Pollock, C., Moore, T., Jacques, A., Burch, J., Gliese, U., Saito, Y., Omoto T., Avanov L., Barrie A., ... Zeuch, M. (2016). Fast plasma investigation for Magnetospheric Multiscale. Space Sci. Rev., 199(1-4), 331-406. https://doi.org/10.1007/s11214-016-0245-4

Priest, E., and Forbes, T. (2000). Magnetic Reconnection: MHD Theory and Applications (pp. 1-45). Cambridge, New York: Cambridge Univ. Press.

Russell, C. T., and McPherron, R. L. (1973). The magnetotail and substorms. Space Sci. Rev., 15(2-3), 205-266. https://doi.org/10.1007/BF00169321

Russell, C. T., Anderson, B. J., Baumjohann, W., Bromund, K. R., Dearborn, D., Fischer, D., Le, G., Leinweber, H. K., Leneman, D., ... Richter, I. (2016). The magnetospheric multiscale magnetometers. Space Sci. Rev., 199(1-4), 189-256. https://doi.org/10.1007/s11214-014-0057-3

Samson, J. C., and Olson, J. V. (1980). Some comments on the descriptions of the polarization states of waves. Geophys. J. R. Astron. Soc., 61(1), 115-129. https://doi.org/10.1111/j.1365-246X.1980.tb04308.x

Sonnerup, B. U. Ö., and Scheible, M. (1998). Minimum and maximum variance analysis. In G. Paschmann, et al. (Eds.), Analysis Methods for Multi-Spacecraft
Data (pp. 185-215). Bern, Switzerland: European Space Agency.

Tang, X. W., Cattell, C., Dombeck, J., Dai, L., Wilson, L. B., Breneman, A., and Hupach, A. (2013). THEMIS observations of the magnetopause electron diffusion region: Large amplitude waves and heated electrons. Geophys. Res. Lett., 40(12), 2884-2890. https://doi.org/10.1002/grl.50565

Torbert, R. B., Russell, C. T., Magnes, W., Ergun, R. E., Lindqvist, P. A., LeContel, O. Vaith, H., Macri, J., Myers, S., ... Lappalainen, K. (2016). The fields instrument suite on MMS: Scientific objectives, measurements, and data products. Space Sci. Rev., 199(1-4), 105-135. https://doi.org/10.1007/s11214-014-01098

Treumann, R. A., and Baumjohann, W. (2015). Spontaneous magnetic reconnection. Collisionless reconnection and its potential astrophysical relevance. Astron. Astrophys. Rev., 23(1), 4. https://doi.org/10.1007/s00159015-0087-1

Vasyliunas, V. M. (1975). Theoretical models of magnetic field line merging. Rev. Geophys., 13, 303-336. https://doi.org/10.1029/RG013i001p00303

Wei, X. H., Cao, J. B., Zhou, G. C., Santolík, O., Rème, H., Dandouras, I., CornilleauWehrlin, N., Lucek, E., Carr, C. M., and Fazakerley A. (2007). Cluster observations of waves in the whistler frequency range associated with magnetic reconnection in the Earths magnetotail. J. Geophys. Res. Space Phys., 112, A10225. https://doi.org/10.1029/2006JA011771

Wilder, F. D., Ergun, R. E., Goodrich, K. A., Goldman, M. V., Newman, D. L., Malaspina, D. M., Jaynes, A. N., Schwartz, S. J., Trattner, K. J., ... Holmes J. C. (2016). Observations of whistler mode waves with nonlinear parallel electric fields near the dayside magnetic reconnection separatrix by the Magnetospheric Multiscale mission. Geophys. Res. Lett., 43(12), 5909-5917. https://doi.org/10.1002/2016GL069473

Yamada, M., Kulsrud, R., and Ji, H. T. (2010). Magnetic reconnection. Rev. Mod. Phys., 82(1), 603-664. https://doi.org/10.1103/RevModPhys.82.603

Zhou, M., Li, H., Deng, X., Huang, S., Pang, Y., Yuan, Z., Xu, X., and Tang R. (2014). Characteristic distribution and possible roles of waves around the lower hybrid frequency in the magnetotail reconnection region. J. Geophys. Res. Space Phys., 119, 8228-8242. https://doi.org/10.1002/2014JA019978 